\title{
Differences in loading patterns between fast walking and jogging at the same speed in male adults
}

\author{
Ruoyi Li ${ }^{1,2}$, Hao Liu', Mengyuan Guo ${ }^{1}$, Jitka Badurova ${ }^{3}$, Luming Yang ${ }^{1,2^{*}}$ and Haojun Fan ${ }^{2}$
}

\begin{abstract}
Fast walking and jogging are two common exercises for people to maintain health in daily life. But the differences in loading patterns of fast walking and jogging are still unclear. The purpose of this study was to compare loading patterns in fast walking and jogging at the same speed, and to identify how differences in foot mechanics influence plantar pressure distribution between the two modes of gait. Totally, 49 healthy males participated in this study. Data of pressure parameters, including maximum force (MF), peak pressure (PP), contact area (CA), force-time integral (FTI), were recorded by Pedar-X insole plantar pressure measurement system in participants' fast walking and jogging process at $7 \mathrm{~km} / \mathrm{h}$. A Load transfer analysis method was used to quantify the plantar load transference from fast walking to jogging. The results showed that MF, PP and CA increased in metatarsal regions and midfoot regions while decreased in toes regions and heel during jogging when compared with fast walking. FTI decreased in all foot regions during jogging compared to fast walking. Under the effects of spring mechanics and the varus of rearfoot during jogging, fast walking and jogging reveal different loading patterns. Compared jogging to fast walking, load transferred as follow: 1) in transverse direction, load transferred from lateral foot to medial foot in metatarsal regions and midfoot regions, 2) in longitudinal direction, load transferred from toes to the metatarsal, and from heel to the metatarsal and midfoot. These results also provide suggestions for footwear designs.
\end{abstract}

Keywords: Loading patterns, Fast walking, Jogging, Load transfer

\section{Introduction}

Fast walking and jogging are both popular forms of exercise for humans to maintain health as their moderate exercise intensity health [1-4]. Fast walking is determined on the basis of natural walking [5]. The walking speed is improved to increase the exercise intensity for calorie consumption [3]. Jogging is sub-classified from running by the velocity. In recreational runners, jogging typically occurring between the speeds of 5-10 mph [6]. The jogging speed can overlap with the fast walking speed. Both fast walking and jogging consist of cyclical subsequent

\footnotetext{
*Correspondence: ylm|l1982@126.com

'National Engineering Laboratory for Clean Technology of Leather

Manufacture, Sichuan University, Chengdu 610065, China

${ }^{2}$ Key Laboratory of Leather Chemistry and Engineering, Sichuan University,

Chengdu 610065, China

Full list of author information is available at the end of the article
}

phases of swing and support [7]. However, fast walking and jogging gait differ at that the former has a double limb support phase while the latter has a float phase instead [6].

As for fast walking and jogging, few studies have compared the two types of daily exercise. While the plantar pressure distribution of walking and running has been compared by many researchers [8-10]. Gerych et al. indicated that peak pressure and maximum force significantly increased in all foot regions during running, and contact area significantly increased in midfoot [10]. Chuckpaiwong et al. compared the in-shoe plantar pressure of walking and running, and found that peak pressure and maximum force significantly increased during running in foot regions while the force-time integral was significantly decreased in the rearfoot, lateral midfoot, 
middle forefoot, and lateral forefoot [8]. However, these previous studies were equivocal because the speed as a variable was not controlled, which was higher in running than in walking. It has been proved that speed could affect plantar pressure both in walking and running [11-13]. Thus, it was difficult to distinguish whether the plantar pressure distribution difference was produced by changing the gait pattern, by the variation in locomotor speed or both factors. Therefore, this study confined fast walking and jogging to the same speed, aiming to eliminate the influence of locomotor speed. As plantar pressure significantly increased in all foot regions during running in the above studies, we hypothesized that plantar pressure would have a great increase during jogging when compared with fast walking.

In this study, a load transfer analysis method was used to identify how the load transferred from fast walking to running. Previous studies had used algorithm and diagram to identify how the load transferred on foot in different situation [14, 15]. However, a similar approach had not been applied in the comparison of fast walking and jogging. The use of this load analysis method could promote a better understanding of the differences on foot loading patterns between fast walking and jogging. It has been reported that walking uses an inverted pendulum model, while running uses a mass-spring model which can exchanges kinetic and energy very differently. Muscles and tendons sequentially store elastic strain energy, and then release the energy through recoil during the stance phase $[16,17]$. As the component of the spring mechanism, the longitudinal arch (LA) play a key role in the store-release energy process on foot $[18,19]$. Here we make a hypothesis that the main difference in the mechanisms of fast walking and jogging was on the function of foot arch.

Therefore, the purpose of this study was to compare loading patterns in fast walking and jogging at the same speed, and to identify how differences in foot mechanics influence plantar pressure distribution between the two modes of gait.

\section{Methods}

\subsection{Participants}

In total, 49 healthy males (age $22.8 \pm 1.7$ years, body mass $65.3 \pm 7.8 \mathrm{~kg}$, height $172.1 \pm 3.8 \mathrm{~cm}$ ), without known neurologic or lower extremity orthopedic pathology or any biomechanical abnormality that may affect gait participated in this study. Informed consent was obtained from each participant. The study was approved by the Ethics Committee of Sichuan University.

Plantar pressure parameters were measured by Novel Pedar-X in-shoe system (Novel, Munich, Germany) at a frequency of $50 \mathrm{~Hz}$. Each pressure insole contained 99 capacitive sensors which were calibrated prior to data collection. The insole was flexible so that it could easily be placed into the bottom of the shoe. All participants were sized and fitted with a pair of test shoes (Nike Free 5.0, Nike, America) to counter a possible bias of shoe type or shoe construction influencing the subjects' walking and running style. The shoe size a US size 8 . The shoes were purchased solely for Pedar data collection and had not been used for any other purpose. The participants were requested to wear the same socks provided during the test. The treadmill (King Smith T211\&T221, China) consisted of a walking surface of $1220 * 420 \mathrm{~mm}$ and could be set to a range of speed from $0.0 \mathrm{~km} / \mathrm{h}$ to16.0 $\mathrm{km} / \mathrm{h}$ accurately.

\subsection{Speed selects}

In recreational runners, jogging typically occurring between the speeds of 5-10 mph [6]. But during the pre-test in the participants, it was difficult to continue walking when the treadmill was set higher than $7 \mathrm{~km} / \mathrm{h}$. Also, it has reported that when the jogging speed was too slow, it would differ from typical running by its more characteristic vertical and "bouncy" running style [9]. Therefore, to avoid the vertical and "bouncy" running style, $7 \mathrm{~km} / \mathrm{h}$ as the highest fast walking speed for most participants can achieve was selected as the test speed for this study.

\subsection{Procedure}

Plantar pressure parameters were recorded as participants walked and ran on the treadmill at $7 \mathrm{~km} / \mathrm{h}$ with no slope wearing the same socks and test shoes. Data of pressure parameters, including maximum force (MF), peak pressure $(\mathrm{PP})$, contact area $(\mathrm{CA})$ and force-time integral (FTI), were recorded by Pedar-X insole plantar pressure measurement system. Prior to testing, all the participants had been informed about the procedures. A warmup was instructed that included regular stretches as well as walking or running at a preferred speed, giving participants time to familiarize themselves with the treadmill and test shoes. After that, treadmill settings were adjusted to $7 \mathrm{~km} / \mathrm{h}$. Data were collected for $20 \mathrm{~s}$ when the participant walked on the treadmill naturally. And then the participant was instructed to change the locomotion into jogging on the treadmill. When the participant jogging naturally, data would be collected for at least $20 \mathrm{~s}$. Approximately one-minute rest was provided during trials if needed.

\subsection{Statistical analysis}

For the analysis of plantar pressure parameters, the foot was divided into eight anatomical regions (Fig. 1): big toe (BT), toes2-5 (T2-5), medial metatarsal (MM), central metatarsal $(\mathrm{CM})$, lateral metatarsals (LM), medial midfoot (MMF), lateral midfoot (LMF) and heel (H). As all participants were right leg dominant, only the right foot of each participant was analyzed [20]. For each 


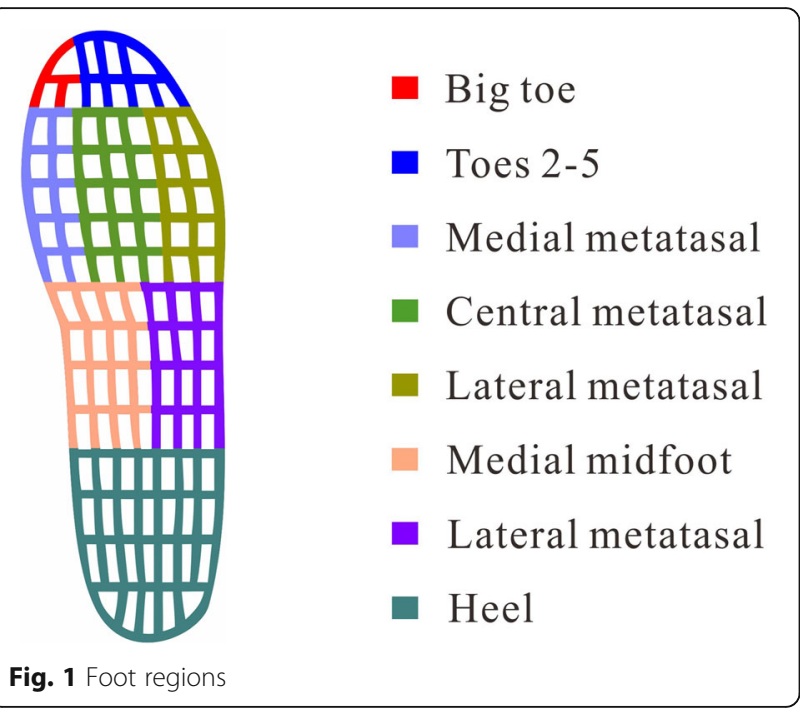

participant, the mean plantar pressure parameters were calculated based on five successful consecutive steps, chosen in the middle of each trial from $20 \mathrm{~s}$ of the collected data. Statistical analysis was conducted by SPSS 17.0. A Paired-Sample $T$ Test was used to determine the main effects of the experimental conditions. The value $p<0.05$ was perceived as significant for statistical analysis.

\subsection{Load transfer analysis method}

A load transfer analysis method was improved which based on the previous study $[14,15]$ to quantify the plantar load transference from fast walking to jogging. Since FTI is the total load in a certain region of the foot which indicates the duration of contact so that the overall load in that region can be fully described, the FTI was calculated for each region [15]. FTI was then normalized into relative FTI for the contrast between participants. Relative FTI was used to assessed the inter-regional load transfer.

$$
\text { Relative FTI }(\%)=\frac{F T I(\text { foot region })}{\sum F T I(\text { foot region })} \times 100 \%
$$

Load transference is divided into two parts: 1) transference in longitudinal direction and 2) transference in transverse direction, in order to observe the transference respectively. Certain rules for the calculation are formulated, as follows:

1. Four levels are defined following the anatomical structure along the longitudinal direction: toes (Level 1, BT and T2-5), metatarsal (Level 2, MM, CM and LM), midfoot (Level 3, MMF and LMF) and heel (Level 4, H). The values in each foot region are difference value of relative FTI between the two gait modes (relative FTI of jogging minus that of fast walking). A negative value indicates the relative FTI of jogging is lower than that of fast walking (green color regions), and vice versa for a positive value (yellow color regions).

2. Load transfer within each level from high value regions to low value regions in transverse (medialateral) direction. Load transfer occurs between adjacent anatomical regions first, and then between the further regions. After the transfer, the relative FTI values in each region at the same level tend to be equal (even in negative values). After the transfer, the altered values of relative FTI are shown in red color. The values on the arrows was the transfer values from one region where the arrow begins to another region where the arrow ends.

3. After the within-level transfer, load transfers through levels in longitudinal (anterior-posterior) direction. Load transfer occurs between adjacent levels first, and then between the further levels. After the transfer, the altered values of relative FTI are exhibited in red color, which are generally zero.

\section{Results}

The results showed that fast walking and jogging had different loading patterns. Compared with fast walking, MF, PP and CA increased in metatarsal and midfoot regions while decreased in toes and heel regions during jogging. Plantar pressure measurements are shown in Fig. 2. In contrast to fast walking, MF significantly increased during jogging in MM, CM, LM, MMF, LMF, and significantly decreased in $\mathrm{BT}, \mathrm{T} 2-5, \mathrm{H}$. The greatest MF concentrated on $\mathrm{H}$ in both fast walking and jogging. Furthermore, the peak value of MF on $\mathrm{H}$ in fast walking was much higher than that in jogging. PP significantly increased during jogging in CM, LM, MMF, LMF, and significantly decreased in BT, T2-5, H compared with fast walking. Significant greater contact area was found in MM, CM, LM, MMF, LMF, and significant lower contact area was found in $\mathrm{T} 2-5, \mathrm{H}$ during jogging than fast walking. Compared the FTI values with fast walking, FTI significantly decreased during jogging in all foot regions. $\mathrm{BT}, \mathrm{MM}$ and $\mathrm{CM}$ were the main loading regions during both fast walking and jogging (Table 1). Meanwhile, $\mathrm{H}$ also took a great proportion of plantar loading during fast walking.

Figure 3 quantifies the plantar load transference from fast walking to jogging. Difference values of relative FTI (\%) between fast walking and jogging at $7 \mathrm{~km} / \mathrm{h}$ are indicated in Table 1. Compared jogging to fast walking, load transferred from BT to T2-5, from LM to MM and CM, and from LMF to MMF in the transverse direction, showing a trend of transferring load from lateral foot to medial foot in metatarsal regions and midfoot regions. In the longitudinal direction, load transferred from level 1 to level 2, and from level 4 to level 2 and level 3, 


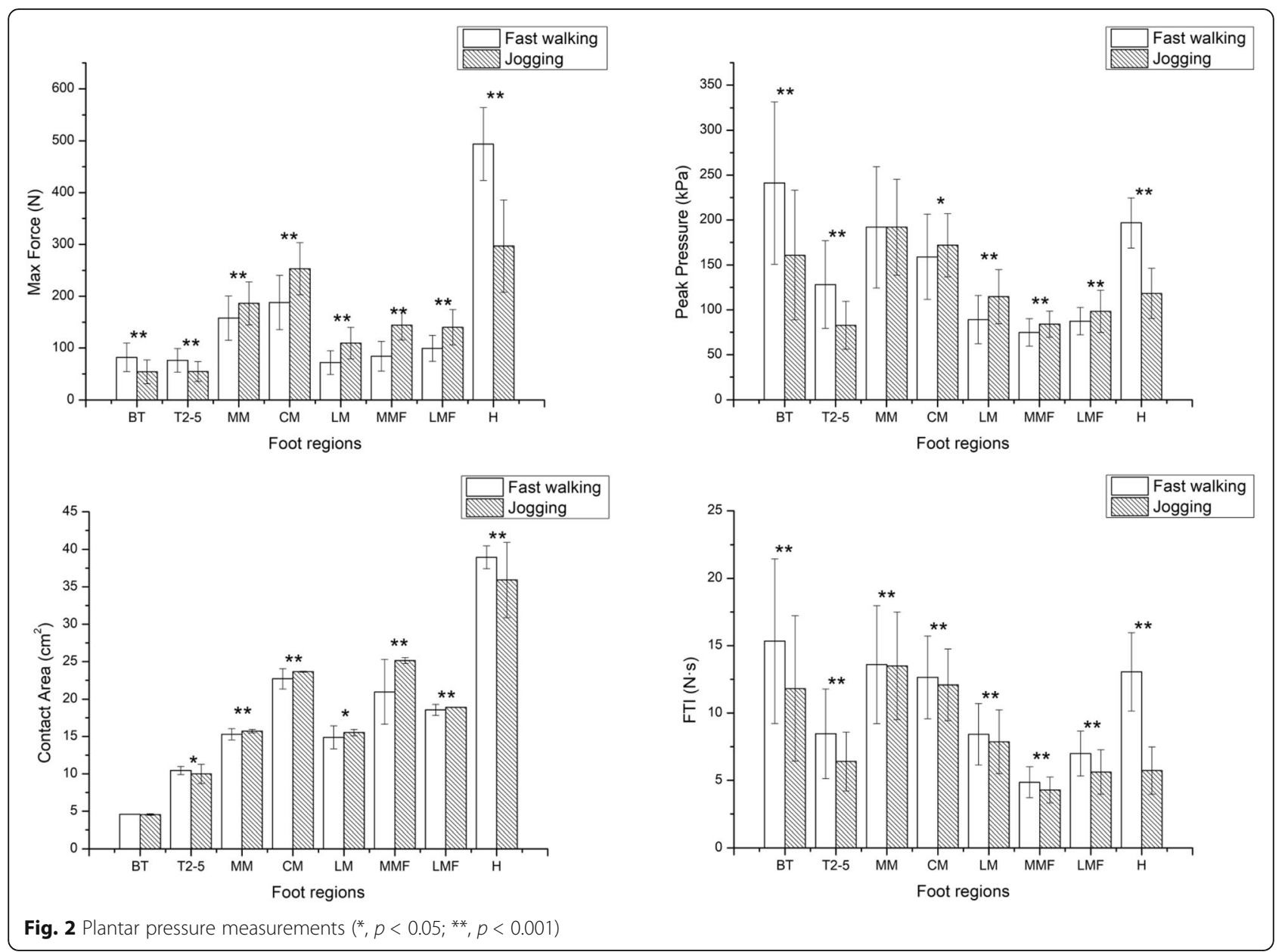

showing that load transferred from toes to the metatarsal, and from heel to the metatarsal and midfoot.

\section{Discussion}

Our first hypothesis was confirmed that plantar pressure had a great increase during jogging when compared with fast walking, but the increase only occurred in metatarsal regions and midfoot regions. MF and PP increased in metatarsal and midfoot regions while decreased in toes and heel regions. These results are different with

Table 1 Relative FTI (\%) in fast walking and jogging at $7 \mathrm{~km} / \mathrm{h}$

\begin{tabular}{llll}
\hline Foot regions & Fast walking & Jogging & Diff. \\
\hline BT & 18.4 & 17.6 & 0.8 \\
T2-5 & 10.2 & 9.5 & 0.7 \\
MM & 16.3 & 20.1 & -3.8 \\
CM & 15.2 & 18.0 & -2.8 \\
LM & 10.1 & 11.7 & -1.6 \\
MMF & 5.8 & 6.4 & -0.6 \\
LMF & 8.4 & 8.3 & 0.1 \\
H & 15.7 & 8.5 & 7.2 \\
\hline
\end{tabular}

previous studies which indicate that MF and PP increase in all of the foot regions during running $[8,10]$. Because that locomotion speed could affect the plantar pressures [11-13] which confuse the effect of changing gait pattern in previous studies. Therefore, speed controlling should be considered into the comparison of different movement modes to eliminate the influence of speeds.

Even at the same speed, fast walking is differentiated from jogging in that the former has a period of double limb support which includes the initial contact phase, loading response phase and pre-swing phase during the fast walking gait [6]. In the double limb support period, both feet are in contact with the ground at the same time. While in the jogging gait, only 1 foot is in physical contact with the ground in these phases. Additionally, there is a float phase that neither foot was in contact with the ground during the jogging gait. Therefore, the foot support was stronger to hold the body and drive to float during jogging than fast walking, which could result in higher impact on the foot than that in fast walking. This provides an explanation that MF and PP increased during jogging than fast walking in this study. Compared to fast walking, MF and PP increased in metatarsal 


\section{Load transference from fast walking to jogging}

Level 1

Level 2

Level 3

Level 4
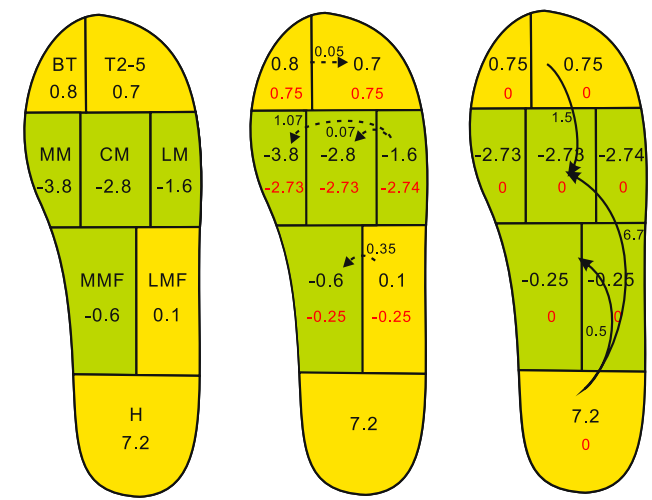

$\rightarrow$ Transverse load transference

$\longrightarrow$ Longitudinal load transference

Fig. 3 Load transference from fast walking to jogging

regions and midfoot regions while decreased in toes and heel regions during jogging. This could be caused by the concentration of foot loading on metatarsal and midfoot regions compared jogging to fast walking.

When encumbered with load, the longitudinal arch (LA) lengthen and lower, subsequently recoiling as the load is removed [21]. Compared with fast walking, the compressive deformation in LA was larger because of the higher force during jogging in metatarsal and midfoot regions. Hence, CA of metatarsal regions and midfoot regions increased during jogging. The increase of $\mathrm{CA}$ in these regions during jogging can help relief the pressure in these foot regions. The consistent increase of $\mathrm{PP}$ with an accompanied increase in MF during jogging compared with fast walking implied that the increase of contact area was not enough to relief the increased force.

In previous studies, peak forces concentrate on forefoot and occur at the end of the stance phase [22, 23]. However, due to the relatively lower speed in the present study, the peak MF occurred at the initial contact phase during jogging which was in accordance with fast walking. At the initial contact phase during fast walking and jogging in this study, the heel strike generated acute force on the heel, which caused the result that the greatest MF concentrated on $\mathrm{H}$ in both fast walking and jogging.

Overall, to the aspect of plantar pressures, the risk of injuries during jogging could be lower than that in fast walking. As the greatest-MF region during both fast walking and jogging, heel had a significantly decrease in MF from $493.9 \mathrm{~N}$ to $296.7 \mathrm{~N}$ during jogging than that in fast walking. While the increase amount of MF in metatarsal regions and midfoot regions was not that much. As the two of the greatest PP regions during fast walking, $\mathrm{BT}$ and $\mathrm{H}$ significant decreased during jogging. So that $\mathrm{MM}$ became the greatest $\mathrm{PP}$ region during jogging where do not have significant difference between fast walking and jogging. The great decrease of PP and MF in toes and heel regions could help reduce the risk of related injuries, such as foot pain [24] and stress fracture [25]. Furthermore, to the best of our knowledge, few shoes were designed especially for fast walking in daily exercise. As fast walking has become more popular, it is suggested that shoes for fast walking should be designed based on the specific plantar pressures during fast walking to reduce the potential risk of injuries. Besides of the protection on metatarsal and heel in running shoes, shoes designed for fast walking should also provide protects in the big toe because of the highest PP in this region.

FTI decreased in all foot regions during jogging compared to fast walking. Since FTI is the total load in a certain region of the foot which indicates the duration of contact, both force and ground contact time can affect the FTI values. Additionally, due to plantar force increased in many foot regions during jogging, the decrease of FTI mainly resulted from the reduction of ground contact time. During fast walking, participants shows longer step length to catch the fast speed than jogging. Compared with fast walking, higher cadence is shown during jogging, which indicate that the time spent on one gait decreased. Furthermore, previous studies have indicated that the ratio of stance time to swing time was approximately 62:38 during walking, while in recreational running the typical ratio was approximately 35:65 [6]. The decreased of stance phase proportion on jogging gait further contributed to lower contact time and lower FTI during jogging.

Walking and running are two distinct gait modes which are different in the energy exchange and mechanics [6, 26-29]. Walking uses the pendular mechanics which can exchange forward kinetic energy for gravitational potential energy during the stance phase. While 
running acts as a mass-spring mechanics in which tendons and ligaments in the compliant lower limb store elastic strain energy and then release the energy through recoil during the stance phase (Fig. 4, a) [16, 17]. As the component of the spring mechanism, the longitudinal arch could maintain flexibility to adapt to alterations in surface and loading demand, as well as providing stiffness to complete the forward propulsion [30]. During fast walking, the less collapse of LA than jogging caused that the bearing of foot load were considerably completed by toes and heel that toes and heel took a large proportion of foot loading (44.3\%). Due to the stance phase of the gait shortens from about $60 \%$ of the gait cycle to as little as $30 \%$ during running, the considerably reduced stance phase causes increased demands on the foot. Therefore, the spring mechanism of foot arch become active (Fig. 4, b). During stance, LA collapses to absorb loading forces as elastic strain energy, and then passive elastic recoil which contribute to the generation of propulsion. As the component of the LA, metatarsal and midfoot suffered more proportion of foot loading during jogging. This also caused the fact that load transferred from toes to the metatarsal, and from heel to the metatarsal and midfoot in the longitudinal direction to engage the spring mechanism to work effectively during jogging. The second hypothesis that the main difference in the mechanisms of fast walking and jogging was on the function of foot arch was then confirmed.

Further, the LA is consisted of lateral longitudinal arch (LLA) and medial longitudinal arch (MLA). Actually, the MLA has a more elastic structure and play a major role in the spring mechanism than the lateral longitudinal arch [18, 31, 32]. Therefore, the activation of MLA during jogging could cause that foot loading more concentrate in the MLA than LLA than that in fast walking. Since foot loading more concentrated in the medial longitudinal arch during jogging, the transfer of foot loading from lateral foot to medial foot (LM $\rightarrow \mathrm{MM}$ and CM,
LMF $\rightarrow$ MMF) was revealed. Additionally, running is different from walking in what is known as "runner's varus" [6]. When the lateral calcaneus contacts at heel strike, shod runners show greater rearfoot varus compared to walking. At initial contact, shod runners typically show a $4^{\circ}-8^{\circ}$ of varum of the calcaneus [33]. The varus of the rearfoot also contributed to the load transfer which from lateral foot to medial foot during jogging compared with fast walking.

Limitations still exist in this study. A potential limitation might be the use of a treadmill. The treadmill could control the speed accurately during the test, but running on the treadmill was associated with lower plantar pressures in foot regions [34]. It was not equivalent to the measurements of the ground. Another limitation was that this study was conducted in healthy males and it was unknown whether similar findings would be achieved in highly trained athletes, injured participants, old people or children where gait parameters would likely vary.

\section{Conclusion}

The function of foot arch plays a key role on the difference of mechanisms on foot between fast walking and jogging. Under the effects of spring mechanics and the varus of rearfoot during jogging, load transferred from lateral foot to medial foot in metatarsal regions and midfoot regions in transverse direction, and transferred from toes to the metatarsal, and from heel to the metatarsal and midfoot in longitudinal direction. As PP had great increases in toes and heel regions during fast walking than that in jogging, the risk of injuries in these regions would be higher during fast walking. Since fast walking has become more popular, we suggest that the shoes designed for fast walking should be designed based on the specific plantar pressures during fast walking to reduce the potential risk of injuries. Besides of the protection on metatarsal and heel in running shoes, fast walking shoes should also provide protects in the big toe because of the highest PP in this region.
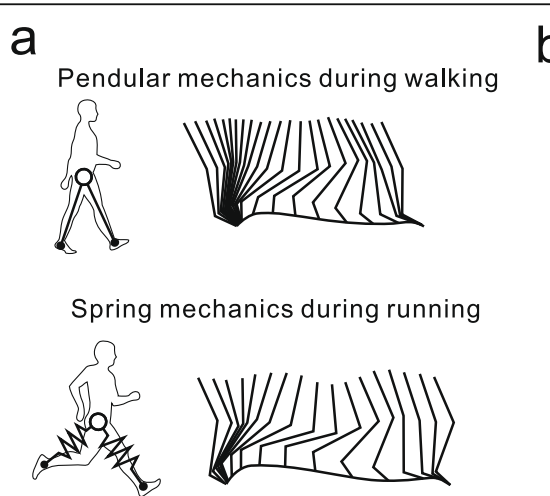

b

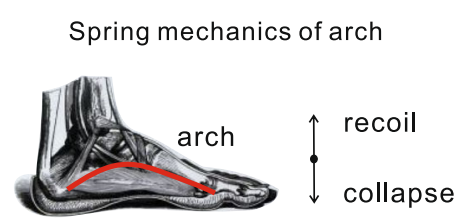

Fig. 4 a) The different mechanics of walking and running (During walking, the leg is relatively straight. During running, human flex the knees more than that in walking). b) Spring mechanics of arch 


\section{Acknowledgements}

Not applicable.

\section{Authors' contributions}

R.L., J. B., L.Y. and H.F. designed the experiment; R.L., H.L. and M.G. carried out the experiment; H.L. and M.G. analyzed experimental data; R.L. and H.L. wrote the manuscript; J. B., L.Y. and H.F. reviewed and improved the manuscript. All authors read and approved the final manuscript.

\section{Authors' information}

Not applicable.

\section{Funding}

This work was supported by National Natural Science Foundation of China, grant number: 11502154.

\section{Availability of data and materials}

The datasets used and analyzed during the current study are available from the corresponding author on reasonable request.

\section{Competing interests}

The authors declare that they have no competing interests.

\section{Author details}

${ }^{1}$ National Engineering Laboratory for Clean Technology of Leather Manufacture, Sichuan University, Chengdu 610065, China. ${ }^{2}$ Key Laboratory of Leather Chemistry and Engineering, Sichuan University, Chengdu 610065,

China. ${ }^{3}$ Tomas Bata University, 76001 Zlin, Czech Republic.

\section{Received: 27 October 2019 Accepted: 30 March 2020}

\section{Published online: 04 May 2020}

\section{References}

1. Ho - -J, Hou Y-Y, Yang C-H, Wu W-L, Chen S-K, Guo L-Y. Comparison of plantar pressure distribution between different speed and incline during treadmill jogging. J Sports Sci Med. 2010.

2. Tsatalas T, Giakas G, Spyropoulos G, Sideris V, Lazaridis S, Kotzamanidis C, et al. The effects of eccentric exercise-induced muscle damage on running kinematics at different speeds. J Sports Sci. 2013;31(3):288-98.

3. Wang WF, Lien WC, Liu CY, Yang CY. Study on tripping risks in fast walking through cadence-controlled gait analysis. J Healthc Eng. 2018:2018:2723178.

4. Chuhong Ma YQ, Tian X. A dynamic observation on college Students' cardiopulmonary load in fitness walking and jogging. J Beijing Sport Univ. 2009:32(5):96-8.

5. Deguchi K, Enishi T, Sato N, Miura H, Fujinaka Y, Matsuhisa M, et al. Acute effect of fast walking on postprandial blood glucose control in type 2 diabetes. Diabetol Int. 2016;7(2):119-23.

6. Lohman EB 3rd, Balan Sackiriyas KS, Swen RW. A comparison of the spatiotemporal parameters, kinematics, and biomechanics between shod, unshod, and minimally supported running as compared to walking. Phys Ther Sport. 2011;12(4):151-63.

7. Saibene FMA. Biomechanical and physiological aspects of legged locomotion in humans. Europ J App Physio. 2003;88:297-316.

8. Chuckpaiwong B, Nunley JA, Mall NA, Queen RM. The effect of foot type on in-shoe plantar pressure during walking and running. Gait Posture. 2008; 28(3):405-11.

9. Keller TS, Weisberger AM, Ray JL, Hasan SS, Shiavi RG, Spengler DM Relationship between vertical ground reaction force and speed during walking, slow jogging, and running. Clin Biomech. 1996:11(5):253-9.

10. Gerych D, Tvrznik A, Prokesova EVA, Nemeckova Z, Jelen K. Analysis of peak pressure, maximal force, and contact area changes during walking and running with conventional and shock-absorbing insoles in the combat boots of the Czech Army. J Mech Med Biol. 2013;13(02):1350042.

11. Chung MJ, Wang MJ. Gender and walking speed effects on plantar pressure distribution for adults aged 20-60 years. Ergonomics. 2012;55(2):194-200

12. Cooper DM, Leissring SK, Kernozek TW. Plantar loading and foot-strike pattern changes with speed during barefoot running in those with a natural rearfoot strike pattern while shod. Foot. 2015:25(2):89-96.

13. Fourchet F, Kelly L, Horobeanu C, Loepelt H, Taiar R, Millet GP. Comparison of plantar pressure distribution in adolescent runners at low vs. high running velocity. Gait Posture. 2012;35(4):685-7.
14. Bus SA, van Deursen RW, Kanade RV, Wissink M, Manning EA, van Baal JG, et al. Plantar pressure relief in the diabetic foot using forefoot offloading shoes. Gait Posture. 2009;29(4):618-22.

15. Hu M, Zhou N, Xu B, Chen W, Wu J, Zhou J. The mechanism of force transference in feet of children ages two to six. Gait Posture. 2017;54:15-9.

16. Claire T, HHPH F, Van Strien C, Louie M. Mechanism of leg stiffness adjustment for hopping on surfaces of different stiffnesses. J Appl Physiol. 1998;85(3):1044-55.

17. Bramble DL, DE. Endurance running and the evolution of homo. Nature. 2004;432(7015):345-52.

18. Kelly LA, Lichtwark $G$, Cresswell $A G$. Active regulation of longitudinal arch compression and recoil during walking and running. J Royal Soc Interface. 2014;12(102):20141076.

19. Kelly LA, Cresswell AG, Racinais S, Whiteley R, Lichtwark G. Intrinsic foot muscles have the capacity to control deformation of the longitudinal arch. J Royal Soc Interface. 2014;11(93):20131188.

20. Menz HB. Two feet, or one person? Problems associated with statistical analysis of paired data in foot and ankle medicine. Foot. 2004;14(1):2-5.

21. MBB RFK, Bibbyt SR, Kestert RC, Alexander RM. The spring in the arch of the human foot. Nature. 1987:325(8):147-9.

22. Pink M, Perry J, Houglum PA, Devine DJ. LOWER-EXTREMITY RANGE OF MOTION IN THE RECREATIONAL SPORT RUNNER. Am J Sports Med. 1994; 22(4):541-9.

23. Fuglevand J, CFMIM. Ground reaction forces in running: a re-examination. J Biomech. 1987;20(2):147-55.

24. Hill CL, Gill TK, Menz HB, Taylor AW. Prevalence and correlates of foot pain in a population-based study: the north West Adelaide health study. J Foot ankle Res. 2008;1(1):2.

25. Ostlie DK, Simons SM. Tarsal navicular stress fracture in a young athlete: case report with clinical, radiologic, and pathophysiologic correlations. J Am Board Fam Pract. 2001;14(5):381-5.

26. Cappellini G, Ivanenko YP, Poppele RE, Lacquaniti F. Motor patterns in human walking and running. J Neurophysiol. 2006;95(6):3426-37.

27. Dugan SA, Bhat KP. Biomechanics and analysis of running gait. Phys Med Rehabil Clin N Am. 2005:16(3):603-21.

28. Li L, van den Bogert ECH, Caldwell GE, van Emmerik REA, Hamill J. Coordination patterns of walking and running at similar speed and stride frequency. Hum Mov Sci. 1999;18(1):67-85.

29. Sasaki K, Neptune RR. Differences in muscle function during walking and running at the same speed. J Biomech. 2006;39(11):2005-13.

30. Hicks J. The mechanics of the foot. II. The plantar aponeurosis and the arch. J Anat. 1954:88(1):25

31. Birinci T, Demirbas SB. Relationship between the mobility of medial longitudinal arch and postural control. Acta Orthop Traumatol Turc. 2017; 51(3):233-7.

32. Caravaggi P, Matias AB, Taddei UT, Ortolani M, Leardini A, Sacco ICN. Reliability of medial-longitudinal-arch measures for skin-markers based kinematic analysis. J Biomech. 2019:88:180-5.

33. Williams KR. Biomechanics of running. Exerc Sport Sci Rev. 1985;13:389-441.

34. Hong Y, Wang L, Li JX, Zhou JH. Comparison of plantar loads during treadmill and overground running. J Sci Med Sport. 2012;15(6):554-60.

\section{Publisher's Note}

Springer Nature remains neutral with regard to jurisdictional claims in published maps and institutional affiliations. 